\title{
In vitro blastocyst development of post-thaw vitrified bovine oocytes
}

\author{
D. J. Dutta, Hiramoni Dev and Himangshu Raj \\ Department of Veterinary Physiology, \\ Faculty of Veterinary Science, Assam Agricultural University, Khanapara, Guwahati-781022, Assam, India \\ Corresponding author: D.J.Dutta, E-mail: duttadj@hotmail.com \\ Received: 26-04-2013, Revised: 13-06-2013, Accepted: 13-06-2013, Published online: 08-08-2013
}

doi: $10.14202 /$ vetworld.2013.730-733

How to cite this article: Dutta DJ, Dev H and Raj H (2013) In vitro blastocyst development of post-thaw vitrified bovine oocytes, Veterinary World 6(10): 730-733.

\begin{abstract}
Aim: To evaluate the developmental competence of post-thaw vitrified bovine cumulus-oocyte complexes (COCs) in vitro.

Materials and Methods: A total of 129 COCs were cryopreserved using vitrification solution comprising of $15 \%$ ethylene glycol (EG) + 15\% dimethyl sulfoxide (DMSO) + 0.6 M sucrose in medium TCM-199 with 10\% FBS. Immediately, within a minute they are plunged into liquid nitrogen using $0.25 \mathrm{ml}$ straws. Thawing was made with a step wise dilution method. Postthaw normal vitrified and non-vitrified oocytes were subjected to in vitro maturation and in vitro fertilization.

Results: Post-thaw survival percentage of vitrified oocytes was $88.37 \%$ and maturation performance of vitrified oocytes on the basis of cumulus expansion was $81.58 \%$ as compared to non-vitrified control $93.85 \%$. The in vitro fertilization performance of vitrified oocytes was $49.46 \%$ as compared to the non-vitrified ones $(63.11 \%)$. Similarly, blastocyst formation of vitrified oocytes was $21.74 \%$ as compared to $32.47 \%$ in non-vitrified oocytes.
\end{abstract}

Conclusion: Vitrification of immature bovine oocytes using 7.5\% EG $+7.5 \%$ DMSO for equilibration and $15 \% \mathrm{EG}+15 \%$ $\mathrm{DMSO}+0.6 \mathrm{M}$ sucrose as vitrification solution yielded better in vitro fertilization and blastocyst formation rate.

Keywords: cumulus-oocyte complexes, in vitro fertilization, in vitro maturation, oocytes, vitrification

\section{I ntroduction}

Retrieval of a higher number of competent oocytes for in vitro maturation and in vitro fertilization (IVM - IVF) to obtain superior transferable bovine embryos coupled with development of freezing technique through vitrification will entail more productivity from the non descript animals. Oocyte cryopreservation is of paramount importance for assisted reproductive technologies (ART). Unique characteristics of mammalian oocytes in respect of permeability of cryoprotectants and water through plasma membrane make them vulnerable for conventional freezing protocols as compared to embryo freezing. Recent advances in freezing techniques like vitrification procedure with modifications in equilibration time, concentration of cryoprotectants, volume and dilution protocol resulted in a higher survival of different stages of oocytes to maturation and fertilization in vitro [1]. The vitrification procedure for freezing technique of oocytes may be more advantageous as compared to conventional freezing protocol. Lesser impact of cryoprotectants as regard to oocytes status with further development in vitro creates new frontiers in ART [2]. Vitrification of immature bovine oocytes using glass micropipettes under normal or super-cooled $\mathrm{LN}_{2}$ resulted in viable blastocysts and live calves following in vitro embryo production [3]. Although oocyte

Copyright: The authors. This article is an open access article licensed under the terms of the Creative Commons Attribution License (http://creativecommons.org/licenses/by/2.0) which permits unrestricted use, distribution and reproduction in any medium, provided the work is properly cited. competence with vitrification procedure seems to improve, additional experiments are required to ascertain its efficacy in oocytes towards its developmental competence in vitro.

Hence the present study was conducted to evaluate the developmental competence of bovine immature oocytes post vitrification.

\section{Materials and Methods}

Oocyte recovery: Cattle ovaries were collected from slaughter house and within $1 \frac{1}{2}-2$ hours, they are processed as per the routine standard protocols. Oocytes were aspirated from 3 to $8 \mathrm{~mm}$ ovarian follicles with medium containing TCM-199 and supplemented with $200 \mathrm{mM} \mathrm{L}$-glutamine solution, $0.4 \%$ BSA and antibiotics. The cumulus-oocyte complexes (COCs) were categorised and morphologically evaluated under stereo zoom microscope [4]. Homogenous and compact COCs were washed four times in holding media (Modified TCM-199, 200mM L-glutamine solution, $10 \%$ FBS, 0.8M Sodium pyruvate and $50 \mu \mathrm{g} / \mathrm{ml}$ Gentamicin and 50 $\mu \mathrm{M}$ Cysteamine) by gentle pipetting and were subjected to cryopreservation by vitrification.

Vitrification and thawing: Two vitrification solutions were prepared in media consisting of TCM-199 with $10 \%$ FBS. Vitrification solution I (VS I) consisted of $7.5 \%$ ethylene glycol (EG) $+7.5 \%$ dimethyl sulfoxide (DMSO) and vitrification solution II (VS II) consisted of $15 \% \mathrm{EG}+15 \% \mathrm{DMSO}+0.6 \mathrm{M}$ sucrose. The immature bovine oocytes with cumulus cells were 
Table-1. Effect of cryoprotectants exposed / vitrified immature oocytes on survival percentagr and maturation in vitro

\begin{tabular}{lcccccc}
\hline Group & No. of COCs & \multicolumn{2}{c}{ Post thaw survivability / recovery performance } & & \multicolumn{2}{c}{ Maturation performance on the basis of cumulus expansion } \\
\cline { 3 - 4 } & & No. & $\%$ & IVM & $\%$ \\
\hline Non-vitrified & 130 & 130 & 100.00 & 122 & $93.85^{\mathrm{a}}$ \\
Vitrified & 129 & 114 & 88.37 & 93 & $81.58^{\mathrm{b}}$ \\
\hline
\end{tabular}

Values in maturation performance column with different superscripts differ significantly. Chi square test $(P<0.05)$

Table-2. In vitro fertilization performances of vitrified and non vitrified bovine immature oocytes.

\begin{tabular}{|c|c|c|c|c|c|c|c|}
\hline \multirow[t]{2}{*}{ Group } & \multirow[t]{2}{*}{ No. of oocytes } & \multicolumn{2}{|c|}{ In vitro fertilization performance } & \multicolumn{4}{|c|}{ Cleavage performance } \\
\hline & & No. & $\%$ & $2-4$ cell $\%$ & $8-16$ cell $\%$ & Morula \% & Blastocysts \% \\
\hline Non-vitrified & 122 & 77 & $63.11^{\mathrm{a}}$ & $77(100)$ & $47(61.03)$ & $25(32.47)$ & $25(32.47)$ \\
\hline Vitrified & 93 & 46 & $49.46^{b}$ & $45(97.83)$ & $22(47.83)$ & $10(21.74)$ & $10(21.74)$ \\
\hline
\end{tabular}

Values in in vitro fertilization performance column with different superscripts differ significantly. Chi square test $(P<0.05)$

exposed to VS I for equilibration upto 3 minutes followed by 25-30 seconds in VS II at room temperature $\left(22-25^{\circ} \mathrm{C}\right)$. The oocytes in VS II were immediately loaded into a $0.25 \mathrm{ml}$ French straw preloaded with 0.6 $\mathrm{M}$ sucrose in holding medium with air gap in between and plunged into Liquid Nitrogen $\left(\mathrm{LN}_{2}\right)$. The straws were stored for a period of 7 days and then thawed in $37^{\circ} \mathrm{C}$ water bath for 30 seconds. After immersion in the water bath, oocytes were gradually rehydrated in sucrose solution. Oocytes were kept into the medium containing $0.6 \mathrm{M}$ of sucrose in basic solution for 1 minute. Then they are transferred successively into holding medium in stepwise dilution pattern containing $0.3 \mathrm{M}$ and $0.15 \mathrm{M}$ of sucrose for one minute in each. Following rehydration, oocytes were washed three times in holding medium. Morphological integrity of post thaw vitrified oocytes was assessed under inverted phase contrast microscope [4]. Oocytes having fragmented zona pellucida and absence of cytoplasmic contents were not considered. The remaining morphologically normal post thaw oocytes were taken for IVM. Freshly collected COCs were separately used for in vitro maturation and kept as control.

In vitro maturation: The fresh or post-thaw vitrified normal oocytes were matured in Modified TCM-199, $200 \mathrm{mM}$ L-glutamine solution, $10 \%$ FBS, $0.8 \mathrm{M}$ Sodium pyruvate and $50 \mu \mathrm{g} / \mathrm{ml}$ Gentamicin and $50 \mu \mathrm{M}$ Cysteamine supplemented with p-FSH $(5 \mu \mathrm{g} / \mathrm{ml}), 10 \%$ $\mathrm{v} / \mathrm{v}$ follicular fluid, $1 \mu \mathrm{g} / \mathrm{ml} 17-\beta$ estradiol at $38.5^{\circ} \mathrm{C}$ in a humidified atmosphere of $5 \% \mathrm{CO}_{2}$ for 24 hours. For confirmation of maturation after 24 hours the oocytes were evaluated for morphological changes and in vitro maturation performance under stereo zoom microscope. The oocytes with an intact zona pellucida, plasma membrane and homogenous cytoplasm were considered as morphologically normal in the study. In vitro maturation performance was assessed on the basis of expansion of cumulus cells surrounding the homogenous oocytes [5].

In-vitro fertilization: For in vitro fertilization, frozen bovine semen (2 straws each) was prepared for capacitation with swim-up technique using B.O. washing medium [6]. After washing, spermatozoa were added to B.O. fertilization medium to make a final concentration of $2 \times 10^{6} \mathrm{sperm} / \mathrm{ml}$. In vitro matured oocytes of both the vitrified and non vitrified groups were co-incubated with spermatozoa in B.O. fertilization medium at $38.5^{\circ} \mathrm{C}, 5 \% \mathrm{CO}_{2}$ in air and saturated humidity for 20-22 hrs. The expected zygotes were washed in culture (IVC) medium (viz., mCR2aa containing 5\%FBS and supplemented with $2 \%$ essential amino acids (v/v), $1 \%$ non essential amino acids (v/v), $1 \% \alpha$ - glutamic acid, $0.3 \%$ BSA and 0.05 $\mu \mathrm{g} / \mathrm{ml}$ gentamicin sulphate). Then zygotes were placed into IVC droplets and covered with mineral oil and incubated at $38.5^{\circ} \mathrm{C}$ under $5 \% \mathrm{CO}_{2}$ in humidified air. After $48 \mathrm{hrs}$ culture the cleavage up to 2-8 cells were recorded. Subsequently culture was continued for further development. Embryos in each group were observed under a microscope every $24 \mathrm{hrs}$ following insemination. Development to 2 cell stage was assessed at $48 \mathrm{hrs}$ after insemination and subsequently cultured for 7 days to evaluate the blastocysts formation. Medium was replaced with fresh medium after every 48 hrs of culture. The study was carried out on different days with replicates of 10 .

Statistical analysis: The data were compiled and the performance of maturation and in vitro fertilization between vitrified and non vitrified groups were compared by chi-square test.

\section{Results and Discussion}

In the present study, a total of 259 good quality bovine COCs were the subject of the experiment. Of which, 129 COCs were subjected to vitrification and the rest were non-vitrified and served as control. The performance in respect of post-thaw survival rates and in vitro maturation on the basis of cumulus cell expansion was more than $80 \%$ respectively. In comparison the non vitrified COCs group, the study demonstrated $93.85 \%$ in vitro maturation performances (Table-1). The vitrification thus had minimal effect on the survival rates and the ability of the oocyte to mature in vitro in the present study. High proportions of bovine COCs retain their post-thaw morphology after a short exposure to high concentration of permeating cryoprotectants namely EG and DMSO with sucrose 
using $0.25 \mathrm{ml}$ straws for freezing. Previous studies on vitrified cattle oocytes had recorded the morphological survival rates between 65 to $94 \%[7,8]$. The sensitivity of bovine oocytes to cryo injury and consequent survival has been well described with variable results. The factors primarily responsible for cell damage are the type of cryoprotectants, concentration, equilibration time, cooling and warming procedures [9]. The maturation rate primarily depends on quality of oocytes, media ingredients and amount and incubation environment. Cooling the Germinal Vesicle (GV) stage bovine oocytes had no effect on the nuclear maturation or fertilization. The nuclear material is membrane bound; thus there is a very low risk of any chromosomal damage as compared to that with dividing cells such as within an embryo or maturing oocytes. Hurtt et al. [10] compared viability of immature and mature bovine oocytes vitrified in Ethylene Glycol based solution and recorded $60 \% \& 70 \%$ nuclear and cytoplasmic maturation rate. Faster membrane penetration of ethylene glycol make it as an ideal cryoprotectant combined with nonpenetrating cryoprotectants like sucrose that act as a stabilizer, minimizing the affect of high concentration of ethylene glycol [11].

The subsequent in vitro fertilization performance of vitrified post-thaw in vitro matured oocytes in the present study was recorded as $49.46 \%$ with $21.74 \%$ of blastocyst formation. The results are in agreement with the findings of Vajta et al. [12] and Papis et al. [13] who used vitrified matured bovine oocytes. The improved success rate was attributed to the increased cooling rate during oocyte vitrification and to the step wise dilution method. Earlier reports show that after thawing using the four step dilution method with 2.5 minutes interval the vitrified oocytes developed to the two cells (71$100 \%$ ), four cells (71-93\%), eight cells (46-71\%) and blastocyst (23-36\%) stages [14]. Step wise dilution might be helpful to reduce osmotic injury of vitrified oocytes after thawing. The cryopreservation capacity of oocytes at the GV stage is lower as compared to metaphase I through metaphase II stages of oocytes have been well documented [15, 16]. However, notably well adapted vitrification program could lessen the differences in the capacity of in vitro development of different maturational stages of oocytes following cryopreservation $[17,18]$.

\section{Conclusions}

Effect of suitable vitrification procedures i.e. using 7.5\% EG+ 7.5\% DMSO for equilibration and $15 \% \mathrm{EG}+15 \% \mathrm{DMSO}+0.6 \mathrm{M}$ sucrose as vitrification solution, on immature bovine oocyte yielded better in vitro oocyte growth and development competence.

\section{Authors' contributions}

All authors contributed equally in this study.

\section{Acknowledgements}

The authors are thankful to the Department of Biotechnology (DBT), Govt. of India for their financial support (Grant no: BT/PR13290/AAQ/01/419/2009 Date: $10^{\text {th }}$ September 2010) towards smooth conduct of this research.

\section{Competing interests}

The authors declare that they have no competing interests.

\section{References}

1. Rienzi, L., Romano, S., Albricci, L., Maggiulli, R., Capalbo, A., Baroni, E., Colamaria, S., Sapienza, F., and Ubaldi, F. (2010) Embryo development of fresh versus vitrified metaphase II oocytes after ICSI: a prospective randomized sibling-oocyte study. Hum Reprod., 25:66-73.

2. Prentice-Biensch, J. R., Singh, J., Mapletoft, R. J., and Anzar, M. (2012) Vitrification of immature bovine cumulus-oocyte complexes: effects of cryoprotectants, the vitrification procedure and warming time on cleavage and embryo development. Reproductive Biology and Endocrinology, 10:73.

3. Vieira, A.D., Forell, F., Feltrin, C., and Rodrigues, J.L. (2008) Calves born after transfer of vitrified bovine in vitroproduced blastocysts derived from vitrified immature oocytes. Reprod Domest Anim. 43 (3):314-8.

4. Dutta, D.J., Sarmah, B.K., and Sarmah, B.C. (1998) Morphological evaluation of caprine oocytes following vitrification. Indian Vet. J., 75 11: 1017-1018.

5. Bols,P.E.J., Jorsen, E.P.A., Goovaerts, I.G.F., Langbeen, A. and Leroy, J.L.M.R. (2012) High throughput non-invassive oocyte quality assessment: the search continues. Animal Reproduction. 9(3): 420-425.

6. Brackett, B.G. and Oliphant, G. (1975) Capacitation of rabbit spermatozoa in vitro. Biol. Reprod., 12: 260-274.

7. Hou, Y.P., Dai, Y.P., Zhu, S.E., Zhu, H.B., Wu, T.Y., Gong, G.C., Wang, H.P., Wang, L.L., Liu, Y., Li, R., Wan, R. and Li, N. (2005) Bovine oocytes vitrified by the open pulled straw method and used for somatic cell cloning supported development to term. Theriogenology, 64(6):1381-91.

8. Anchamparuthy, V.M., Dhali, A., Lott, W.M., Pearson, R.E. and Gwazdauskas, F.C.(2009) Vitrification of bovine oocytes: implications of follicular size and sire on the rates of embryonic development. JAssist Reprod Genet., 26(11-12): 613-619.

9. Diez, C., Duque, P., Gomez, E., Hidalgo, C.O., Tamargo, C., Rodríguez, A., Fernández, L., de la Varga, S., Fernández, A., Facal, N. and Carbajo, M. (2005) Bovine oocytes itrification before or after meiotic arrest: effects on ultrastructure and developmental ability. Theriogenology, 64: 317-333.

10. Hurtt, A.E., Landim-Alvarenga, F., Scidel Jr., G.E. and Squires, E.L. (2000) Vitrfication of immature and mature equine and bovine oocytes in an ethylene glycol, ficoll and sucrose solution using open pulled straws. Theriogenology, 54.1: 119-128.

11. Saragusty, J. and Arav, A. (2011) Current progress in oocyte and embryo cryopreservation by slow freezing and vitrification. Reproduction. 141:1-19.

12. Vajta, G., Holm, P., Kuwayama, M., Booth, P.J., Jacobsen, H., Greve, T. and Callesen, H. (1998) Open pulled straw (OPS) vitri? cation: a new way to reduce cryoinjuries of bovine ova and embryos. Mol Reprod Dev., 51:53-58.

13. Papis, K., Shimizu, M. and Izaike, M.(1999) The effect of gentle pre-equilibration on survival and development rates of bovine in vitro matured oocytes vitri?ed in droplets. Theriogenology, 51:173.

14. Hong, S.W., Chung, H.M., Lim, J.M., Ko, J.J., Yoon, T.K., Bill, Yee, and Cha, K.Y. (1999) Improved human oocyte development after vitri?cation: a comparison of thawing methods. Fertility and Sterility, 72(1): 142-146.

15. Schroeder, A.C., Champlin, A.K., Mobraaten, L.E., and Eppig, J.J. (1990) Developmental capacity of mouse oocytes cryopreserved before and after maturation in vitro. J Reprod 
Fertil, 89:43-50.

16. Lim, J.M., Fukui, Y. and Ono, H.(1992) Developmental competence of bovine oocytes frozen at various maturation stages followed by in vitro maturation and fertilization. Theriogenology, 37:351-61.

17. Shirazi, A., Taheri, F., Nazari, H., Norbakhsh-Nia, M., Ahmadi, E. and Heidari B. (2012) Developmental competence of ovine oocyte following vitrification: effect of oocyte developmental stage, cumulus cells, cytoskeleton stabiliser, FBS concentration and equilibration time. Zygote, 1-9.

18. Prentice J. R., Singh J., Dochi O., and Anzar M. (2011) Factors affecting nuclear maturation, cleavage and embryo development of vitrified bovine cumulus-oocytes complex. Theriogenology, 75: 602-609.

$* * * * * * * *$ 